\title{
Negative regulation by thyroid hormone receptor requires an intact coactivator-binding surface
}

\author{
Tania M. Ortiga-Carvalho, ${ }^{1,2}$ Nobuyuki Shibusawa, ${ }^{3}$ Amisra Nikrodhanond, ${ }^{1}$ Karen J. Oliveira, ${ }^{2}$ \\ Danielle S. Machado, ${ }^{1}$ Xiao-Hui Liao, ${ }^{1}$ Ronald N. Cohen, ${ }^{1}$ Samuel Refetoff, ${ }^{1,4}$ \\ and Fredric E. Wondisford 1

\begin{abstract}
1Department of Medicine and Committee on Molecular Metabolism and Nutrition, Pritzker School of Medicine, The University of Chicago, Chicago, Illinois, USA. ${ }^{2}$ Instituto de Biofísica Carlos Chagas Filho, Universidade Federal do Rio de Janeiro, Rio de Janeiro, Brazil. ${ }^{3}$ Department of Medicine and Molecular Science, Gunma University Graduate School of Medicine, Maebashi, Japan. ${ }^{4}$ Department of Pediatrics and the Committee on Genetics, The University of Chicago, Chicago, Illinois, USA.
\end{abstract}

\begin{abstract}
Thyroid hormone (TH) action is mediated by TH receptors (TRs), which are members of the nuclear hormone receptor superfamily. In vitro studies have demonstrated that $T R$ activity is regulated by interactions with corepressor and coactivator proteins (CoRs and CoAs, respectively). TH stimulation is thought to involve dissociation of CoRs and recruitment of CoAs to the liganded TR. In contrast, negative regulation by TH is thought to occur via recruitment of CoRs to the liganded TR. The physiological role of CoAs bound to TRs, however, has yet to be defined. In this study, we used gene-targeting techniques to mutate the TR- $\beta$ locus within its activation function-2 (AF-2) domain (E457A). This mutation was chosen because it completely abolished CoA recruitment in vitro, while preserving normal triiodothyronine $\left(T_{3}\right)$ binding and CoR interactions. As expected, THstimulated gene expression was reduced in homozygous E457A mice. However, these animals also displayed abnormal regulation of the hypothalamic-pituitary-thyroid axis. Serum thyroxine, $T_{3}$, and thyroid-stimulating hormone (TSH) levels and pituitary Tshb mRNA levels were inappropriately elevated compared with those of WT animals, and $L-T_{3}$ treatment failed to suppress serum TSH and pituitary $T s h b$ mRNA levels. Therefore, the AF-2 domain of TR- $\beta$ is required for positive and, paradoxically, for negative regulation by TH in vivo.
\end{abstract}

\section{Introduction}

The synthesis of thyrotropin-releasing hormone (TRH), produced in the hypothalamus, and the $\alpha$ and $\beta$ subunits of thyrotropin (thyroidstimulating hormone $[\mathrm{TSH}])$ in the anterior pituitary is inhibited at the transcriptional level by thyroid hormone $(\mathrm{TH})(1)$. Negative regulation of gene expression by triiodothyronine $\left(T_{3}\right)$ is critical in the control of the hypothalamic-pituitary-thyroid (H-P-T) axis, and this effect is mediated by the $\beta$ isoform of the TH receptor (TR) (2-6).

$\mathrm{TH}$ action is mediated by different isoforms of the TR, which are members of the nuclear receptor superfamily of ligand-modulated transcriptional factors (7). Two genes, by alternative splicing and alternative transcriptional site utilization, express all known ligand-binding TR isoforms, TR- $\alpha 1$, TR- $\beta 1-3$. Mice deficient in either TR- $\alpha$ or TR- $\beta$ isoforms display unique phenotypes, which suggests that different TR isoforms have unique regulatory roles $(4-6,8)$. On genes with a positive TH response element (pTRE) and in the absence of hormone, TR associates with corepressor proteins (CoRs), such as silencing mediator for retinoid and thy-

Nonstandard abbreviations used: AF-2, activation function-2; CoA, coactivator protein; CoR, corepressor protein; Dio1, $5^{\prime}$-deiodinase type I; Gsta, glutathione-S-transferase; H-P-T, hypothalamic-pituitary-thyroid; LoI/PTU diet, low iodine diet containing $0.15 \%$ 5-propyl-2-thiouracil; $\mathrm{L}_{3}$, L-triiodothyronine; MMI, methimazole; NCoR, nuclear CoR; nTRE, negative TRE; pTRE, positive TRE; RIP140, receptor-interacting protein 140; RXR, retinoid X receptor; SMRT, silencing mediator for retinoid and thyroid hormone receptors; SRC-1, steroid receptor CoA- $1 ; \mathrm{T}_{3}$, triiodothyronine; $\mathrm{T}_{4}$, thyroxine; TH, thyroid hormone; TR, TH receptor; TRE, TH response element; TRH, thyrotropin-releasing hormone; TSH, thyroid-stimulating hormone.

Conflict of interest: The authors have declared that no conflict of interest exists.

Citation for this article: J. Clin. Invest. 115:2517-2523 (2005).

doi:10.1172/JCI24109. roid hormone receptors (SMRT) and nuclear $\mathrm{CoR}$ (NCoR), which assemble a complex that inhibits gene transcription. In the presence of ligand, this CoR complex dissociates and is replaced by a coactivator protein (CoA) complex that then activates transcription at the promoter (7). In contrast, at a negative $\mathrm{TH}$ response element (nTRE), activation of transcription occurs in the absence of ligand, when, paradoxically, TR might be expected to be in a complex with CoRs and not CoAs (7).

Several models have been suggested to explain the mechanism of negative regulation by TH. On the TRH and TSH subunit genes, TR DNA binding has also been reported to be necessary for negative regulation by $\mathrm{TH}(9-12)$. Some investigators, however, have suggested models wherein TR DNA binding is not required for negative regulation. In a report on one such model, it was proposed that both the DNA-binding domain of TR- $\beta$ and direct DNA binding of TR within the target gene were not necessary for TR-mediated transrepression (13). This mechanism has been proposed to explain negative regulation of the Tsha promoter, in which an nTRE in the proximal promoter has not been clearly identified $(14,15)$. A DNA binding-independent mechanism similar to that for the nuclear hormone receptor action has also been proposed for the glucocorticoid receptor (16). In contrast, our group has demonstrated, using a welldefined TR DNA-binding P box mutant (GS125), that DNA binding by TR is required for negative regulation both in vitro (17) and in vivo (18). Thus, models of negative regulation must accommodate a TR bound to a target gene in the absence and presence of $\mathrm{TH}$.

Given the importance of DNA binding for negative regulation by $\mathrm{TH}$, we next sought to determine what additional functions of the TR are required. The aim of this study was to create a transgenic 


\section{A}

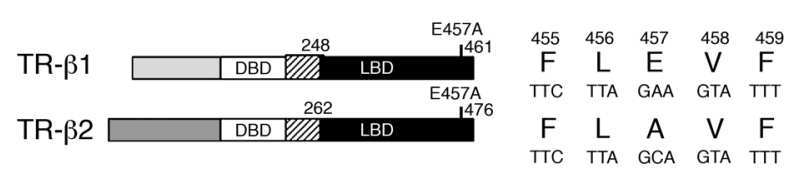

B

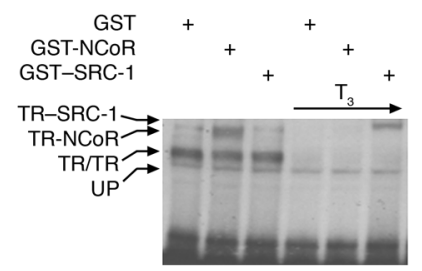

TR- $\beta 2$

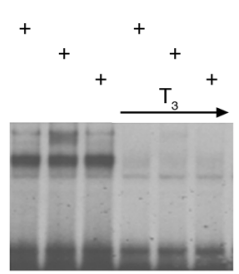

TR- $\beta 2$ (E457A)

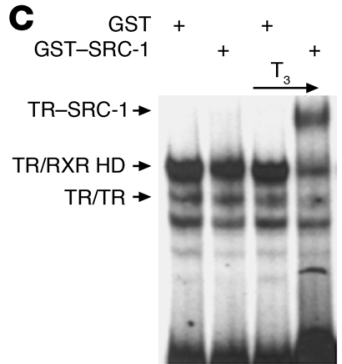

TR- $\beta 2$

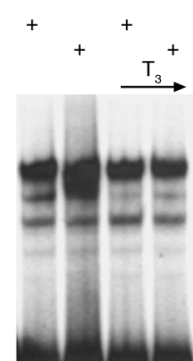

TR-ß2 (E457A)
D

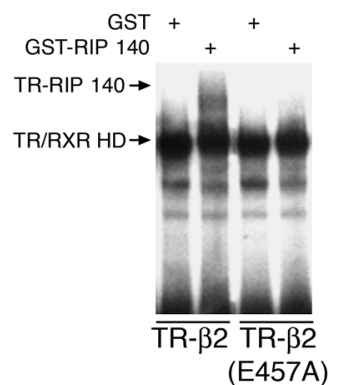

E

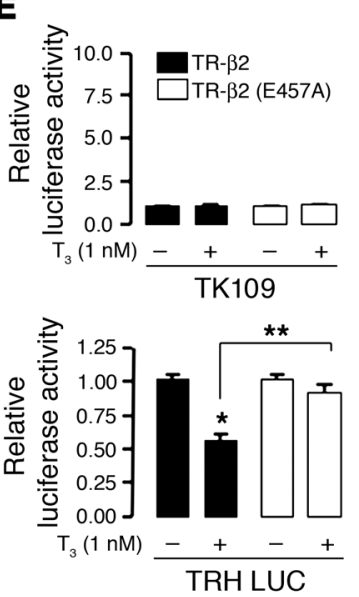

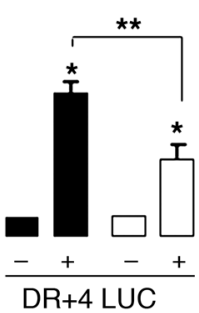

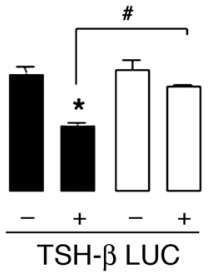

Figure 1

In vitro characterization of TR- $\beta$ (E457A). (A) Schematic representation of the TR- $\beta$ isoforms and the location of the E457A mutation. Amino acid numbers are given for the human mutation in the mouse TR- $\beta$ locus. DBD, DNA-binding domain; LBD, ligand-binding domain. (B and C) Gel mobility shift assay showing that the E457A receptor mutant binds to CoR (NCoR) but not to CoA (SRC-1) protein. RXR was added to all the lanes shown in C. A positive TRE (DR+4 probe) was radiolabeled, and $\mathrm{T}_{3}$ was added at a concentration of $10 \mathrm{nM}$. HD, heterodimer; UP, unprogrammed or non-specific. (D) Gel mobility shift assay showing that the E457A receptor mutant does not bind to RIP140 in the presence of $T_{3}$ on the same radiolabeled $D R+4$ element. $R X R$ and $T_{3}$ were added to all lanes. (E) Effect of mutant TR- $\beta$ (E457A) on positive and negative TREs. Ligand-dependent transcriptional activity of positively regulated reporter gene DR+4 LUC and negatively regulated reporter genes TRH LUC (human TRH -900 to +55 bp) and TSH- $\beta$ LUC (human TSH -1192 to +37 bp). TK 109, a minimal thymidine kinase promoter in pA3Luc, was used as a control. Equal amounts of WT or mutant TR-32-expressing plasmids or empty vector (SG5) were cotransfected into HEK 293 T cells with a luciferase reporter gene. The data are presented as fold change in activity compared with the level in the absence of $T_{3}$. Data represent 5 separate experiments performed in triplicate. ${ }^{*} P<0.001$ vs. no $\mathrm{T}_{3} ;{ }^{* \star} P<0.001 ;{ }^{\#} P<0.01$.

mouse model in which the CoA-binding site on TR- $\beta$ was disrupted, while maintaining CoR and $\mathrm{T}_{3}$ binding activity. Results in the E457A mutant mice demonstrated that an intact CoA-binding surface is important for positive regulation in vivo. Surprisingly, this same domain is also essential for negative regulation of gene expression in the H-P-T axis and liver.

\section{Results}

To address the importance of the CoA-binding surface on TR- $\beta$ in vivo, we utilized a helix 12 mutant of TR- $\beta$ (E457A; Figure $1 \mathrm{~A})$ with normal $\mathrm{T}_{3}$ binding affinity $(19,20)$. When tested in gel mobility shift assays, it displayed normal affinity for both pTREs and CoR (NCoR) but was unable to bind the CoA, steroid receptor CoA-1 (SRC-1), and receptor-interacting protein 140 (RIP140), after $\mathrm{T}_{3}$ treatment (Figure $1, \mathrm{~B}-\mathrm{D})$. Moreover, this mutant was almost completely defective in mediating negative regulation by $\mathrm{TH}$ and was partially impaired in mediating positive gene regulation (Figure 1E).

The E457A mutation was then introduced into the Thrb locus via homologous recombination in ES cells (exon 7, Figure 2A). Homologous recombination in ES clones was confirmed by Southern blot analysis and PCR (data not shown). Chimeric males were derived from 2 targeted ES cell clones and transmitted the mutation through crosses with C57BL/ 6 females; heterozygous mutant mice were interbred. The genotype of $\mathrm{F}_{1}$ and subsequent generations was identified by Southern blot analysis (Figure 2B).

To establish that TR- $\beta$ was mutated in E457A mice, we first examined TR- $\beta$ transcripts by performing RT-PCR analysis on total RNA extracted from adult mouse pituitary tissue of WT (TR- $\left.\beta^{W T / W T}\right)$ and E457A mutant heterozygous (TR- $\beta^{E 457 A / W T}$ ) and homozygous $\left(T R-\beta^{E 457 A / E 457 A}\right)$ animals (Figure $\left.2 \mathrm{C}\right)$ using primers described in Methods. PCR products from TR- $\beta$ (E457A) knock-in mice were the same size as those from WT animals (Figure $2 \mathrm{C}$ ). The presence of E457A mutation was confirmed by subsequent sequencing of the cloned PCR product (Figure 2D). E457A mutant TR- $\beta$ mRNA was expressed at the same level as WT Thrb mRNA, as indicated by real-time PCR analysis of total pituitary RNA (Figure 2E), which indicated that the mutation did not affect expression from the Thrb locus. Moreover, this mutation did not affect Thra expression in the same animals. E457A knock-in mice were born with no gross anatomic or functional abnormalities and were viable through adulthood. Both males and females displayed normal fertility.

Serum thyroxine $\left(T_{4}\right)$ and $T_{3}$ levels were higher in TR- $\beta$ (E457A) knock-in mice, as shown in Figure 3. The TR- $\beta^{E 457 A / E 457 A}$ mice displayed $\mathrm{T}_{3}$ and $\mathrm{T}_{4}$ levels up to 5 times higher than those in WT mice ( $P<0.001$; Figure $3, \mathrm{~A}$ and $\mathrm{B})$. Heterozygous animals displayed a 2 -fold increase in $\mathrm{T}_{4}$ levels compared with those in 
A

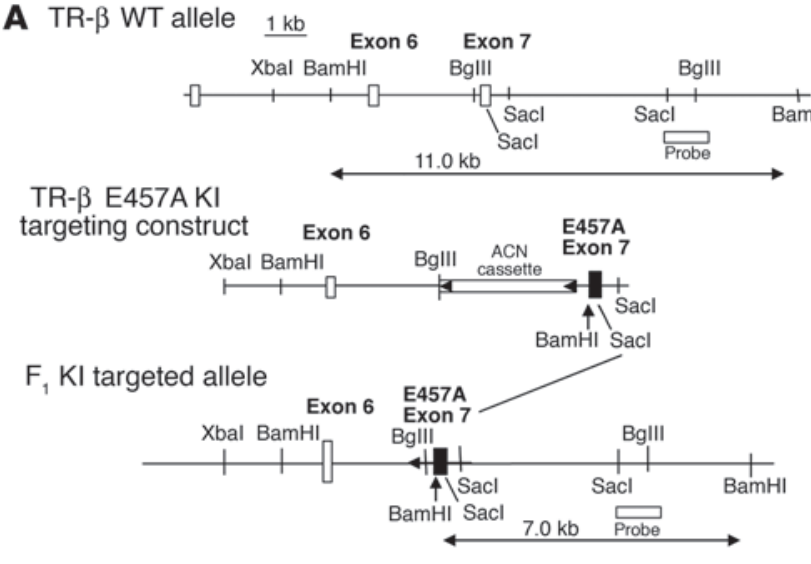

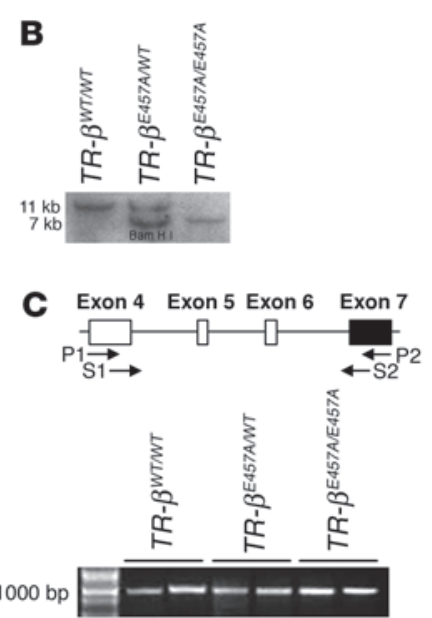

E

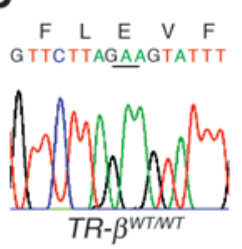

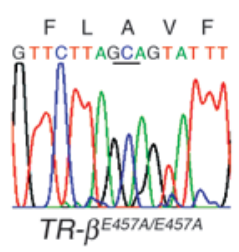

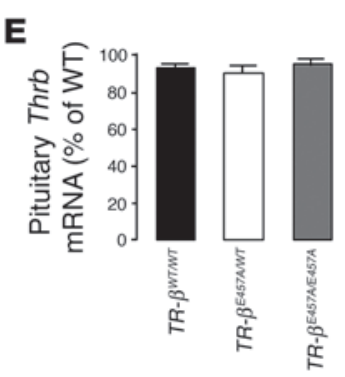

\section{Figure 2}

Characterization of TR- $\beta$ (E457A) mice. (A) Introduction of E457A mutation into the mouse TR- $\beta$ gene by homologous recombination. KI, knock-in. (B) Homologous integration of the targeting vector was confirmed by Southern blot analysis of genomic DNA digested with $\mathrm{BamHI}$ and probed with a probe located outside the targeting vector. (C) RT-PCR of TR- $\beta$ from pituitary RNA using primers described in Methods. A 950bp fragment from the TR- $\beta$ WT/WT, TR- $\beta$ E457A/WT , and TR- $\beta$ E457A/E457A mice was observed. Two different animal tissues from each group were analyzed. (D) DNA sequence of the 950-bp fragment. The mutated amino acid is underlined. (E) Thrb and Thra mRNA levels in the anterior pituitary of TR- $\beta$ WT/WT, TR- $\beta$ E457A/WT, and TR- $\beta$ E457A/E457A mice according to quantitative RT-PCR analysis. Data were normalized for each mRNA level relative to the values for the WT animals $(n=5-8)$.
WT mice $(P<0.001)$; and $\mathrm{T}_{3}$ values were higher than those of control animals, although the difference was not statistically significant (Figure 3B). Similar results were obtained in both male and female mice (data not shown). Basal serum TSH levels were also elevated in the homozygous mice, reaching up to 9 times those observed in WT animals ( $P<0.001$; Figure $3 \mathrm{C})$. Tshb subunit mRNA levels were similarly elevated at baseline in the homozygous animals. Although individual heterozygous animals displayed elevated TSH values, the mean level was not significantly different from that of WT animals (Figure 3C).

Serum TSH and Tshb subunit mRNA levels (Figure 3D) were not suppressed in TR- $\beta^{E 457 A / E 457 A}$ mice, despite elevated circulating TH concentrations, which indicates that they have central TH resistance - resistance at the level of the pituitary and/or hypothalamus. In addition to central TH resistance, the TR- $\beta^{E 457 A / E 457 A}$ and TR- $\beta \beta^{E 457 A / W T}$ mice also displayed peripheral tissue resistance. Glutathione-S-transferase (Gsta) mRNA levels in the liver, which are normally suppressed in the presence of elevated TH levels (21), were elevated in homozygous mutant mice (Figure 3E).

Histological comparison of the thyroid glands in TR $-\beta^{E 457 A / E 457 A}$ mice with those in WT mice revealed an overall enlargement, as predicted from the serum hormone levels (Figure 4). Despite normal body weight, the thyroid weight of TR- $\beta^{E 457 A / E 457 A}$ mice was approximately 3 -fold greater than that of control animals $(P<0.001$; Figure 4). Thyroid weight of heterozygous mice was also increased, but to a lesser extent and was consistent with the small increase in serum TSH concentration found in these animals.

To investigate further the molecular mechanism of abnormal regulation of the H-P-T axis in TR- $\beta^{E 457 A / E 457 A}$ animals, we examined mice in both a TH-deficient and an excess state. After 35 days of a low iodine diet containing $0.15 \%$ 5-propyl-2-thiouracil (LoI/PTU diet) supplemented with methimazole (MMI) in the water, all ani- mals exhibited markedly elevated TSH levels (>15,000 mU/1; Figure $5 \mathrm{~A}$ ) and undetectable serum $\mathrm{T}_{4}$ levels (data not shown). $\mathrm{L}-\mathrm{T}_{3}$ treatment was then administrated by daily subcutaneous injections for 5 days at 3 escalating concentrations. Unlike in control and heterozygous animals, serum TSH and Tshb subunit mRNA levels in TR- $\beta^{E 457 A / E 457 A}$ mice were not suppressed completely even at the highest $\mathrm{T}_{3}$ dose, which indicated markedly reduced sensitivity to negative feedback regulation by TH (Figure 5, A and D). At the end of the middle dose period, TR- $\beta^{E 457 A / W T}$ mice also exhibited elevated TSH levels compared with WT animals, although this difference did not achieve statistical significance. Unlike serum TSH and TSH- $\beta$ subunit mRNA levels, TSH- $\alpha$ subunit RNA levels were suppressed in all 3 groups after administration of the higher dose of $L-T_{3}$ (Figure 5C). However, the degree of suppression in homozygous animals was much less than that observed in WT mice.

To investigate the effects of the E457A mutant in peripheral tissues, we measured the expression of negatively and positively regulated genes in the liver. Gsta was used as a negatively regulated gene in the liver (21). The Gsta expression profile was similar to that of Ts $h b$ : mRNA levels in the TR- $\beta^{E 457 A / E 457 A}$ mice were not suppressed after administration of the highest ${\mathrm{L}-\mathrm{T}_{3}}_{3}$ dose (Figure $6 \mathrm{~A}$ ), which indicated markedly reduced sensitivity to TH inhibition of gene expression. 5' deiodinase type I (Dio1) was used as a positively regulated gene in the liver. Dio1 mRNA levels increased in the $T R-\beta^{W T / W T}$ and TR- $\beta^{E 457 A / W T}$ animals after $\mathrm{L}-\mathrm{T}_{3}$ treatment, although the increase in Dio1 expression was lower in heterozygous animals. In contrast, Dio1 expression in TR- $\beta^{E 457 A / E 457 A}$ mice was insensitive to $\mathrm{L}-\mathrm{T}_{3}$ treatment (Figure 6B).

\section{Discussion}

Negative feedback regulation of the H-P-T axis has been well studied because of its physiological importance. A number of reports 

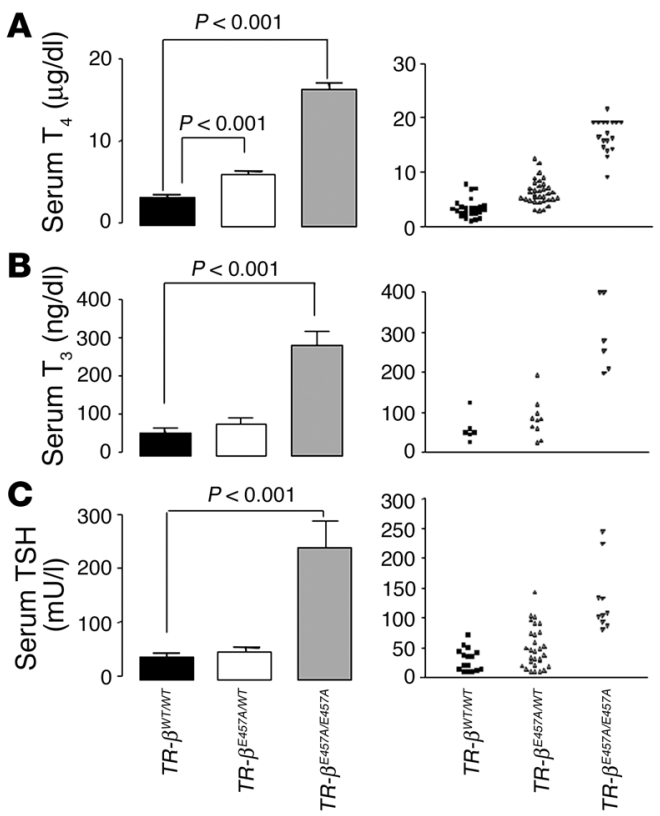

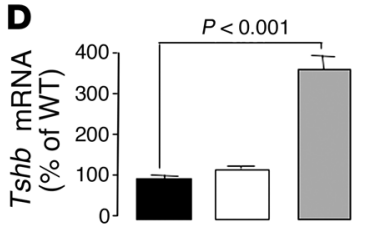

E

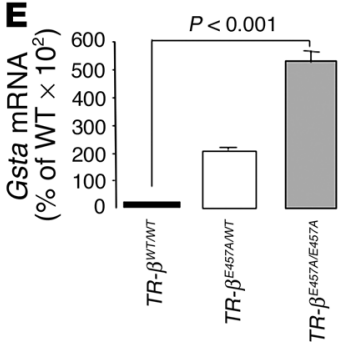

\section{Figure 3}

Analysis of the H-P-T axis of the WT and E457A mutant mice. (A-C) Total serum $\mathrm{T}_{4}, \mathrm{~T}_{3}$, and TSH levels $(n=13-34)$. (D and E) Pituitary Tshb and liver Gsta mRNA levels of TR- $\beta^{W T / W T}, T R-\beta^{E 457 A / W T}$, and $T R-\beta$ E457A/E457A mice according to quantitative RT-PCR analysis. Data were normalized for each mRNA level relative to values for the WT animals $(n=6-10)$. Data are shown as mean \pm SEM. have identified elements responsible for negative regulation and proposed mechanisms and target cofactors based on the traditional "on-DNA" hypothesis $(9,12,22)$ - i.e., TR bound to DNA. Recently, one group has suggested that the conformation of the CoR bound to the TH response element (TRE) determined whether it would be an nTRE or pTRE (23). Alternatively, "off-DNA" models for negative regulation have also been proposed by several groups - i.e., TR not bound to DNA. These models depend on the ability of non-DNA-bound TR to sequester a limiting transcription factor in the absence (nuclear CoR model) or presence (activator protein 1 model) of $\mathrm{T}_{3}(15,24,25)$. These models question, therefore, whether nTREs truly exist.

We mutated the DNA-binding P box domain of TR- $\beta$ in mice (GS125) to critically test the validity of these models (19). Using these animals, we showed that DNA binding by TR on negatively regulated genes was essential for the $\mathrm{T}_{3}$-mediated transcriptional regulation, which indicates that the off-DNA hypothesis is likely not valid in vivo. Of course, it is possible that the TR DNA-binding domain interacts directly with cofactors off-DNA, via a yet-to-be-described mechanism, and that the GS125 mutation blocks this interaction. Given the minimal change to the DNA-binding domain, however, this mechanism seems less likely. Thus, negative regulation by $\mathrm{TH}$, like positive regulation, requires TR binding to target gene elements.

While both negative and positive regulation by $\mathrm{TH}$ require receptor DNA binding, there are fundamental differences in these processes. On positively regulated genes, unliganded TRs occupy pTREs and silence gene expression through association with CoRs, such as $\mathrm{NCoR}$ and SMRT (7). Transcription repression is mediated through the recruitment of $\mathrm{mSin} 3 \mathrm{~A}$ (isoform A of the mammalian $\operatorname{Sin} 3$ protein) and histone deacetylases (26), which compact nucleosomes into a tight and less accessible structure. When $\mathrm{T}_{3}$ binds to the TR, TR homodimers are dissociated, CoRs are released, TR/retinoid X receptor (TR/RXR) heterodimeric binding predominates, and CoAs such as SRC-1 with histone acetyltransferase (HAT) activity are recruited to the TR activation function-2 (AF-2) domain (8).

However, this model cannot explain negative regulation by $\mathrm{TH}$, which is essentially the opposite process. In the absence of $\mathrm{T}_{3}, \mathrm{TR}$ activates gene expression, while in the presence of $\mathrm{T}_{3}$, expression is repressed. The fact that DNA binding is essential for negative regulation indicates 1 of the following possibilities: (a) the same cofactor acts as a coactivator or corepressor on positive versus negative TREs, respectively; or (b) different cofactors are recruited to mediate positive versus negative $T_{3}$ regulation.

The present study shows that the E457A mutation in vivo significantly affects $\mathrm{T}_{3}$ regulation, which suggests a high degree of resistance to TH. Elevated TSH subunit mRNA levels, serum TSH concentrations, and $\mathrm{TH}$ levels demonstrate that regulation of the H-P-T axis is impaired in E457A mutant mice, which indicates that an intact CoA-binding surface is essential for negative feedback regulation of the H-P-T axis in vivo. This defect in negative regulation is more severe than that reported in TR- $\beta-\mathrm{KO}$ animals and

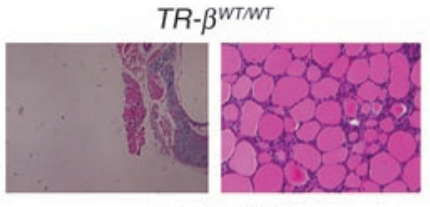

TR- $\beta^{\text {E457AWT }}$

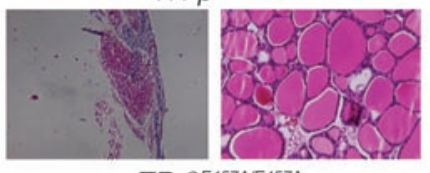

TR- $\beta^{\text {E457AVE457A }}$

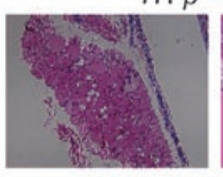

$\times 25$

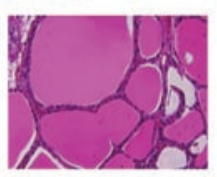

$\times 200$
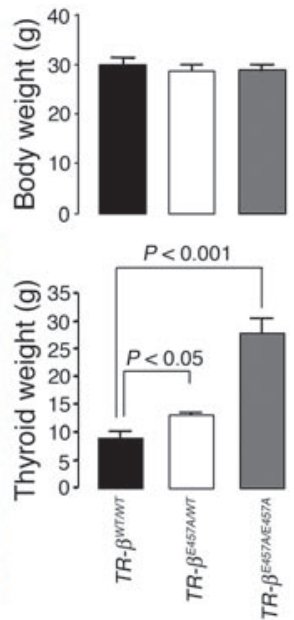

\section{Figure 4}

Analysis of the thyroid gland and body weight of the TR- $\beta^{W T / W T}$, $T R-\beta E 457 A / W T$, and TR- $\beta$ E457A/E457A mice. Six to 12 animals were evaluated in each group, and data are shown as mean \pm SEM. 
A

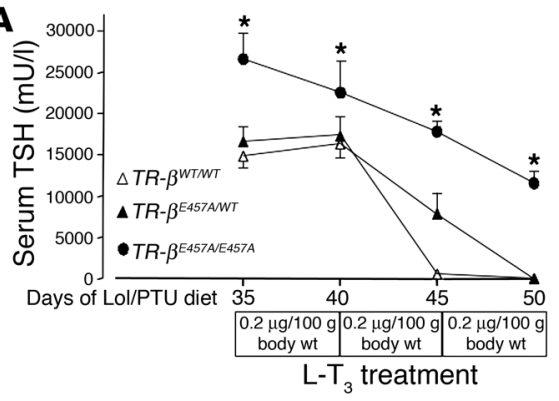

B After Lol/PTU diet

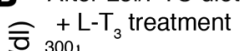

$\widehat{\overline{0}}+\mathrm{L}_{300} \mathrm{~T}_{3}$ treatment
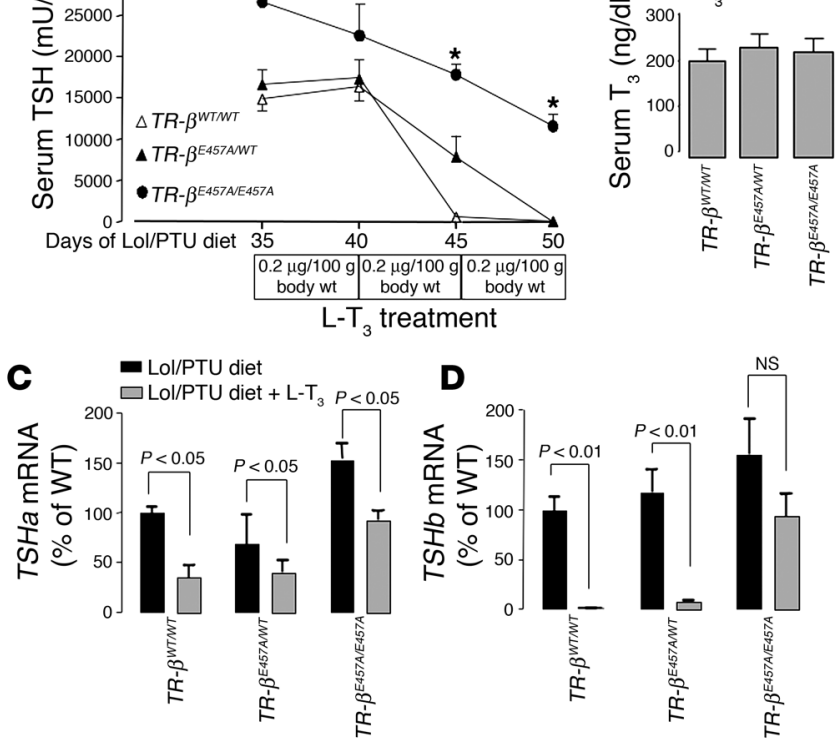

similar to that found in mutant mice expressing ligand-binding mutation of TR- $\beta(27,28)$. Moreover, regulation of Dio1 and Gsta mRNA levels in the liver was also affected in E457A mutant animals, which demonstrates that this mutation impairs both positive and negative regulation by $\mathrm{T}_{3}$ in the same organ.

It was previously reported that the E457A mutation specifically disrupted CoA binding, while preserving normal interactions with CoRs and $\mathrm{T}_{3}$ binding affinity $(19,20,29)$. We have confirmed these properties of the mutant receptor in vitro (Figure 1). In addition, 2 other mutant TRs that bind $T_{3}$ normally and cause resistance to TH (RTH) have been identified $(20,29)$. These mutations in Thrb codon 454 retain normal DNA binding but also exhibit impaired CoR release. Therefore, these mutations are distinct from E457A and cause RTH primarily by a decrease in CoR release as opposed to impaired CoA recruitment. Like the E457A mutation, these mutations are in a highly conserved region of the distal ligand-binding region, termed $\mathrm{AF}-2 . \mathrm{T}_{3}$ receptor binding induces movement of the AF-2 helix or helix 12, displacing CoR proteins and leading to recruitment of $\mathrm{CoA}(30,31)$.

CoAs are proteins that can remodel chromatin via enzymatic modification of histone tails by acetylation or via regulation of transcription at the promoter by interaction with RNA polymerase and general transcription factors (32). The designation of these factors as CoAs was initially predicated on their nuclear localization and their ability to interact with and amplify liganddependent functions of nuclear receptors on TRE-linked reporter genes (33). One of the first CoAs to be cloned was SRC-1, and SRC-1-KO mice have been shown to have resistance to sex hormones (34) as well as to TH (35). However, the lack of SRC-1 function alone would not explain our data. While SRC-1-deficient mice have total serum $\mathrm{T}_{4}, \mathrm{~T}_{3}$, and TSH concentrations 1.5 -fold to 2.5 -fold higher than those in WT animals (35), corresponding values in the E457A mice are 5- to 9-fold higher. In addition to these baseline data, the E457A animals showed a significantly greater impairment in $\mathrm{L}-\mathrm{T}_{3}$-mediated suppression of the H-P-T axis, which indicates a higher degree of resistance to TH in these mice compared with SRC-1-KO animals.

\section{Figure 5}

Effect of TH deficiency and excess on regulation of the H-P-T axis. (A) Serum TSH levels were sequentially determined in $T R-\beta W T / W T$, TR- $\beta$ E457A/WT, and TR- $\beta$ E457A/E457A mice after they had been on the Lol/ PTU diet and given MMI water for 5 weeks and had received the additional treatment with different doses of $\mathrm{L}-\mathrm{T}_{3}$ for 5 days each. ${ }^{*} P<0.01$ vs. WT. (B) Total serum $T_{3}$ levels at the end of the $L-T_{3}$ treatment. (C and D) Tshb and Tsha mRNA levels in the anterior pituitary of TR- $\beta$ WT/WT, $T R-\beta^{E 457 A / W T}$, and TR- $\beta^{E 457 A / E 457 A}$ mice using a quantitative RT-PCR analysis. Data were normalized for each mRNA level relative to values for the WT animals. Five to 10 animals were evaluated in each group, and data are shown as mean \pm SEM.

The response of a tissue to TH is directly dependent on several factors, including the amount of hormone available, the conversion rate of $\mathrm{T}_{4}$ to $\mathrm{T}_{3}$, the number of TRs, and the quantity and nature of transcriptional cofactors available within the cell. We controlled for differences in TH levels by rendering mice hypothyroid and then replacing $\mathrm{T}_{3}$ with escalating $\mathrm{L}-\mathrm{T}_{3}$ doses. Also, TR receptor isoform levels were not different among the animals. So differences seen between groups are directly related to the point mutation and its effect on cofactor binding. The observation that SRC-1-KO mice have a mild resistance to steroid and TH confirmed that SRC-1 functions physiologically as a CoA. Although our data demonstrate that the E457A mutation blocks SRC-1 binding, it must also interfere with other unknown factors to explain the higher resistance to TH seen in the pituitary (Figure 3 and 5) and in the liver (Figure 6) of these mice.

Ligand-dependent recruitment of CoAs would seem counterintuitive to a mechanism of negative regulation, whereby ligand reduces gene expression. Recently, however, Xu et al. (36) have shown that CoA-associated arginine methyltransferase 1 (CARM1) can methylate the P160 CoA class and reduce its HAT activity. In the E457A animals, the absence of SRC-1 binding would prevent methylation by CARM1 and might thereby be a mechanism explaining

A

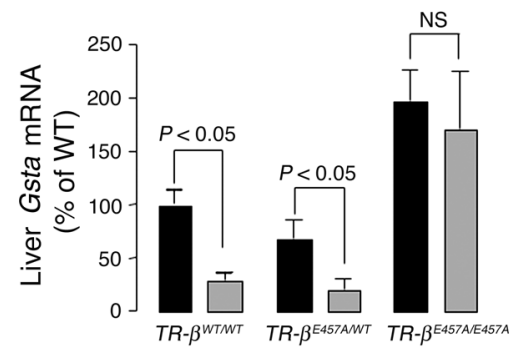

B

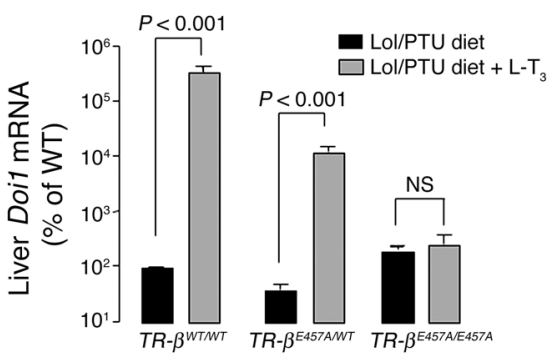

Figure 6

Gsta (A) and Doi1 (B) mRNA levels in the liver of the TR- $\beta^{W T / W T}$, $T R-\beta E 457 A / W T$, and TR- $\beta$ E457A/E457A mice according to quantitative RTPCR analysis. Data were normalized for each mRNA level relative to the values for the WT animals. Five to 10 animals were evaluated in each group, and data are shown as mean \pm SEM. 
the lack of negative regulation in these animals. Alternatively, a unique cofactor could be recruited to the liganded TR resulting in repression by $T_{3}$. At least 2 cofactors have been described that could function in this way: RIP140 and ligand-dependent CoR ( $\mathrm{L}-\mathrm{CoR})(37,38)$. These cofactors are recruited to nuclear hormone receptor AF-2 domains in the presence of ligand; but in contrast to CoAs, these cofactors repress gene transcription. They do so by recruiting either histone deacetylases or a different repressing protein termed C-terminal binding protein $(39,40)$. Here we show that the E457A mutation in the TR- $\beta 2$ prevents the recruitment of RIP140 in vitro, which could explain the inability of $\mathrm{T}_{3}$ to repress TRH and TSH- $\beta$ subunit gene expression in vivo.

In summary, the AF- 2 domain of TR- $\beta$ is required for both positive and negative regulation by TH in vivo. Paradoxically, the AF-2 domain must also function as a ligand-dependent repressor in vivo, given the defect in negative regulation in E457A mice. Further studies will be required to determine whether ligand-dependent CoAs, with modified function, or ligand-dependent CoRs mediate this regulatory effect.

\section{Methods}

\section{Transfections}

Plasmid constructions. A point mutation of the Thrb was introduced by sitedirected mutagenesis in the context of the TR- $\beta 2$ isoform. The E457A mutant TR- $\beta 2$ cDNA was inserted into the PSG5 expression vector at an EcoRI site. A luciferase reporter construct containing a positive TRE $(\mathrm{DR}+4$ LUC) has been previously described (41). Negatively regulated gene reporters have also been described: human TRH -900 to +55 bp (TRH LUC) and human TSH $\beta-1192$ to +37 bp (TSH LUC) (17). TK 109, a minimal thymidine kinase promoter in pA3Luc, was used as a negative control (42).

Cell culture and transient transfection assay. HEK $293 \mathrm{~T}$ cells were maintained in DMEM supplemented with L-glutamine, 10\% FCS, $100 \mu \mathrm{g} / \mathrm{ml}, 0.25 \mu \mathrm{g} / \mathrm{ml}$ streptomycin and amphotericin. Transient transfections were performed in subconfluent 6-well plates; $1.7 \mu \mathrm{g}$ of reporter construct with $0.075 \mu \mathrm{g}$ of receptor expression vector or the same amount of PSG5 vector alone were added to each well using the calcium phosphate technique $(11,17)$. After 15-18 hours, culture medium containing FBS depleted of TH by treatment with anion exchange resin (AG 1-X8, analytical grade; Bio-Rad Laboratories) was added. Twenty-four hours after transfection, $1 \mathrm{nM} \mathrm{T}_{3}$ was added, and 24 hours after transfection, cultures were harvested and assayed for luciferase activity.

Gel mobility shift assay. Gel mobility shift analysis of TR- $\beta 2$ interactions was conducted with nuclear hormone receptor interaction domains of the CoRs $\mathrm{N}-\mathrm{CoR}$ (43) and RIP140 (37) and the CoA SRC-1 (22). WT (TR- $\beta 2)$, mutant TR- $\beta 2$ (E457A), and RXR proteins were derived from in vitro transcription/ translation reactions in rabbit reticulocyte lysate (Promega). Interacting domains of the cofactors were synthesized as Gsta fusion proteins. Fifty nanograms of NCoR or $500 \mathrm{ng}$ of SRC-1 and RIP140 were used in the indicated lanes. Interactions were tested on radiolabeled $\mathrm{DR}+4$ element as previously described (41). In some reactions, $\mathrm{T}_{3}$ was added at a concentration of $10 \mathrm{nM}$.

Generation of E457A mutant knock-in mice. The targeting construct was electroporated into ES cells of the 129 strain, and G418 neomycin-resistant colonies were isolated and expanded. ES cells electroporated with the targeting vector were screened by Southern blot analysis with an external probe. Two ES clones of the construct were then injected into C57BL/6 blastocysts to generate chimeric mice, and chimeric mice were bred with C57BL/ 6 female mice. Chimeric males derived from targeted ES cells clones transmitted the mutation through crosses with C57BL/6 females, and the resulting heterozygous mice were interbred. Genotyping of tail DNA from E457A animals was performed by Southern blot analysis.
Mice were maintained under 12-hour light/12-hour dark cycles (beginning at $6 \mathrm{am} / 6 \mathrm{pm})$. All mice used in these experiments were of the same mixed background strain (129/C57BL/6), and WT animals were littermate controls. All animal experiments were performed according to the NIH Guide for the Care and Use of Laboratory Animals and approved by the Institutional Animal Care and Use Committee of the University of Chicago.

Serum TH and TSH measurements. Serum TH levels (total $\mathrm{T}_{3}$ and $\mathrm{T}_{4}$ ) were measured by solid-phase radioimmunoassay (Coat-A-Count; Diagnostic Products Corp.). Mouse serum TSH levels were measured by a sensitive heterologous radioimmunoassay, as described previously (44). TH deficiency was induced in 8-week-old mice in each of 3 groups - WT (TR- $\left.\beta^{W T / W T}\right)$, heterozygous (TR- $\left.\beta^{E 457 A / W T}\right)$, and homozygous $\left(T R-\beta^{E 457 A / E 457 A}\right)-$ on a LoI/ PTU diet (Harlan Teklad Co.) and 0.05\% MMI in water. After 5 weeks, animals received either vehicle or daily subcutaneous injections of a low $(0.2$ $\mu \mathrm{g} / 100 \mathrm{~g}$ body wt/d), medium $(0.5 \mu \mathrm{g} / 100 \mathrm{~g}$ body wt/d), or high $(1.0 \mu \mathrm{g} / 100$ g body wt/d) dose of L-T 3 (Sigma-Aldrich) for 5 days each. The LoI/PTU diet and MMI in water were given throughout the $L-T_{3}$ treatment period. The animals were sacrificed 24 hours after the last injection of $\mathrm{L}-\mathrm{T}_{3}$.

RNA analysis. Total RNA was extracted using a standard method (TRIZOL Reagent; Invitrogen Corp.). RT and PCR analyses were carried out on $1 \mu \mathrm{g}$ of total pituitary RNA using AMV RT and Tfl DNA polymerase (Access RT-PCR System; Promega). For PCR analysis, 1 pair of primers, 5'-GCCAAGCGGAAGCTTATAGAG-3' (P1) and 5'-ACACAAAGAAACGAAGAAACA-3' (P2), was used to amplify the ligand-binding domain of the TR- $\beta$ (exon 4 to exon 7; Figure 2 C). RT-PCR products from each genotype were analyzed by direct cycle sequencing using the following internal primers: 5'-GCACAAGCCAGAACCCACGGA-3' (S1) and 5'-CAGTCATGCTGCAGGAATGAC-3' (S2).

For quantitative real-time RT-PCR analysis, RT was carried out on $2 \mu \mathrm{g}$ of total pituitary or liver RNA. Real-time RT-PCR analyses were performed in a fluorescent temperature cycler (MyiQ Single-Color Real-Time PCR Detection System; Bio-Rad Laboratories) according to the recommendations of the manufacturer. Briefly, after initial denaturation at $50^{\circ} \mathrm{C}$ for 2 minutes and $95^{\circ} \mathrm{C}$ for 10 minutes, reactions were cycled 40 times using the following parameters for all genes studied: $95^{\circ} \mathrm{C}$ for 15 seconds, $60^{\circ} \mathrm{C}$ for 30 seconds, and $70^{\circ} \mathrm{C}$ for 30 seconds. SYBR Green (Bio-Rad Laboratories) fluorescence was detected at the end of each cycle to monitor the amount of PCR product formed during that cycle. Primers used for the amplification of cDNAs of interest were synthesized by Integrated DNA Technologies Inc. The sequence of the forward and reverse primers, respectively, were: $5^{\prime}$ GTGTATGGGCTGTTGCTTCTCC-3' and 5'-GCACTCCGTATGATTCTCCACTCTG-3' for Tsha; $5^{\prime}$-TCTCGCCGTCCTCCTCTCCGTGCTT-3' and 5'-AGTTGGTTCTGACAGCCTCGTG-3' for Ts $b b$; $5^{\prime}$-CGGCTACCACATCCAAGGAA- $3^{\prime}$ and $5^{\prime}$-GCTGGAATTACCGCGGCT-3' for Rn $18 s$; $5^{\prime}$-GCAATGGCCGGGAAGC-3' and 5'-ACCTGATGCACTCCATTCTGC-3' for Gsta; $5^{\prime}$-AGCCAGCTCTACGCGGC-3' and 5'-CCCTTGTAGCAGATCCTGCC$3^{\prime}$ for Dio1; 5'-TTCTCT CCTTCCTCCCATCCTTT-3' and 5'-GGCTGGAGGGTCTGAGGG-3' for Thra; and 5'-CAGCCTGGGACAAGCAGAA-3' and 5'-TTCAGACATTCCTACCAGCTTCC-3' for Thrb.

We determined relative mRNA levels $(2 \Delta \mathrm{C} t)$ by comparing the PCR cycle threshold $(\mathrm{Ct})$ between groups. We checked the purity of the PCR products by analyzing the melting curves. Each sample was measured in duplicate, and each experiment was repeated at least 3 times. Results are expressed relative to the values for WT expression, which were considered to be equal to $100 \%$.

Histology. Thyroid glands were excised and washed once with PBS and then fixed in $10 \%$ neutral buffered formalin and embedded in paraffin, and $6-\mu \mathrm{m}$-thick section were prepared and stained with $\mathrm{H} \& \mathrm{E}$.

Statistical analysis. Data are reported as mean \pm SEM. One-way ANOVA followed by Student-Newman-Keuls multiple comparisons test was employed for assessment of significance when comparisons were made 
within the same genotype. Two-way ANOVA was employed when mice of different genotypes and treatment were compared (GraphPad Prism version 4.0a; GraphPad Software Inc.). Differences were considered to be significant at $P<0.05$.

\section{Acknowledgments}

This work was supported by grants from the NIH to F.E. Wondisford (DK49126 and DK53036) and the Diabetes Research and Training Center at the University of Chicago (DK20595). The authors would like to thank S. Radovick for her helpful discussions and K. Markan for technical assistance.
Received for publication December 6, 2004, and accepted in revised form May 31, 2005.

Address correspondence to: Fredric E. Wondisford, Division of Metabolism, Departments of Pediatrics and Medicine, Johns Hopkins Medical Institute, 600 Wolf Street/Park 211, Baltimore, Maryland 21287, USA. Phone: (410) 955-6463; Fax: (410) 955-9773; E-mail: fwondisford@jhmi.edu.

Tania M. Ortiga-Carvalho and Nobuyuki Shibusawa are co-first authors.
1. Cohen, R.C., and Wondisford, F.E. 2005. Chemistry and biosynthesis of thyrotropin. In Werner and Ingbar's the thyroid: a fundamental and clinical text. 9th edition. L.E. Braverman and R.D. Utiger, editors. Williams \& Wilkins. Philadelphia, Pennsylvania, USA. $159-175$.

2. Forrest, D., Erway, L.C., Ng, L., Altschuler, R., and Curran, T. 1996. Thyroid hormone receptor beta is essential for development of auditory function. Nat. Genet. 13:354-357.

3. Weiss, R.E., et al. 1997. Thyrotropin regulation by thyroid hormone in thyroid hormone receptor beta-deficient mice. Endocrinology. 138:3624-3629.

4. Abel, E.D., et al. 1999. Divergent roles for thyroid hormone receptor $\beta$ isoforms in the endocrine axis and auditory system. J. Clin. Invest. 104:291-300.

5. Gauthier, K., et al. 1999. Different functions for the thyroid hormone receptors TRalpha and TRbeta in the control of thyroid hormone production and post-natal development EMBO J. 18:623-631.

6. Abel, E.D., Ahima, R., Boel, M.E., Elmquist, J.K., and Wondisford, F.E. 2001. Critical role for thyroid hormone receptor beta2 in the regulation of paraventricular thyrotropin-releasing hormone neurons. J. Clin. Invest. 107:1017-1023.

7. Lazar, M.A. 2003. Thyroid hormone action: a binding contract [review]. J. Clin. Invest. 112:497-499. doi:10.1172/JCI200319479.

8. Flamant, F., and Samarut, J. 2003. Thyroid hormone receptors: lessons from knockout and knock-in mutant mice. Trends Endocrinol. Metab. 14:85-90.

9. Bodenner, D.L., Mroczynski, M.A., Weintraub, B.D., Radovick, S., and Wondisford, F.E. 1991. A detailed functional and structural analysis of a major thyroid hormone inhibitory element in the human thyrotropin beta-subunit gene. J. Biol. Chem. 266:21666-21673.

10. Naar, A.M., et al. 1991. The orientation and spacing of core DNA-binding motifs dictate selective transcriptional responses to three nuclear receptors. Cell. 65:1267-1279.

11. Hollenberg, A.N., Monden, T., and Wondisford, F.E. 1995. Function of nuclear co-repressor protein on thyroid hormone response elements is regulated by the receptor A/B domain. J. Biol. Chem. 270:14274-14280.

12. Sasaki, S., et al. 1999. Ligand-induced recruitment of a histone deacetylase in the negative-feedback regulation of the thyrotropin beta gene. $E M B O J$. 18:5389-5398.

13. Tagami, T., Madison, L.D., Nagaya, T., and Jameson, J.L. 1997. Nuclear receptor corepressors activate rather than suppress basal transcription of genes that are negatively regulated by thyroid hormone. Mol. Cell. Biol. 17:2642-2648.

14. Madison, L.D., Ahlquist, J.A., Rogers, S.D., and Jameson, J.L. 1993. Negative regulation of the glycoprotein hormone alpha gene promoter by thyroid hormone: mutagenesis of a proximal receptor binding site preserves transcriptional repression.
Mol. Cell. Endocrinol. 94:129-136.

15. Tagami, T., Park, Y., and Jameson, J.L. 1999 Mechanisms that mediate negative regulation of the thyroid-stimulating hormone alpha gene by the thyroid hormone receptor. J. Biol. Chem. 274:22345-22353.

16. Bledsoe, R.K., Stewart, E.L., and Pearce, K.H. 2004. Structure and function of the glucocorticoid receptor ligand binding domain. Vitam. Horm. 68:49-91.

17. Shibusawa, N., Hollenberg, A.N., and Wondisford, F.E. 2003. Thyroid hormone receptor DNA binding is required for both positive and negative gene regulation. J. Biol. Chem. 278:723-738.

18. Shibusawa, N., et al. 2003. Thyroid hormone action in the absence of thyroid hormone receptor DNA-binding in vivo. J. Clin. Invest. 112:588-597. doi:10.1172/JCI200318377.

19. Tone, Y., Collingwood, T.N., Adams, M., and Charttejee, V.K. 1994. Functional analysis of a transactivation domain in the thyroid hormone beta receptor. J. Biol. Chem. 269:31157-31161.

20. Tagami, T., Gu, W.X, Peairs, P.T., West, B.L., and Jameson, J.L. 1998 A novel natural mutation in the thyroid hormone receptor defines a dual function domain that exchanges nuclear receptor corepressors and coactivators. Mol. Endocrinol. 12:1888-1902.

21. Sadow, P.M., et al. 2003. Specificity of thyroid hormone receptor subtype and steroid receptor coactivator-1 on thyroid hormone action. Am. J. Physiol. Endocrinol. Metab. 284:E36-E46.

22. Cohen, O., Flynn, T.R., and Wondisford, F.E. 1995. Ligand-dependent antagonism by retinoid $\mathrm{X}$ receptors of inhibitory thyroid hormone response elements. J. Biol. Chem. 9:13899-13905.

23. Berghagen, H., et al. 2002. Corepressor SMRT functions as a coactivator for thyroid hormone receptor T3Ralpha from a negative hormone response element. J. Biol. Chem. 277:49517-49522.

24. Zhang, X.K., Wills, K.N., Husmann, M., Hermann, T., and Pfahl, M. 1991. Novel pathway for thyroid hormone receptor action through interaction with jun and fos oncogene activities. Mol. Cell. Biol. 11:6016-6025

25. Claret, F.X., Hibi, M., Dhut, S., Toda, T., and Karin, M. 1996. A new group of conserved coactivators that increase the specificity of AP-1 transcription factors. Nature. 383:453-457.

26. Pazin, M.J., and Kadonaga, J.T. 1997. What's up and down with histone deacetylation and transcription [review]? Cell. 89:325-328.

27. Kaneshige, M., et al. 2000. Mice with a targeted mutation in the thyroid hormone beta receptor gene exhibit impaired growth and resistance to thyroid hormone. Proc. Natl. Acad. Sci. U. S. A. 97:13209-13214.

28. Hashimoto, K., et al. 2001. An unliganded thyroid hormone receptor causes severe neurological dysfunction. Proc. Natl. Acad. Sci. U. S. A. 98:3998-4003.

29. Collingwood, T.N., et al. 1997. A natural transactivation mutation in the thyroid hormone beta receptor: impaired interaction with putative tran- scriptional mediators. Proc. Natl. Acad. Sci. U. S. A. 94:248-253.

30. Feng, W., et al. 1998. Hormone-dependent coactivator binding to a hydrophobic cleft on nuclear receptors. Science. 280:1747-1749.

31. Marimuthu, A., et al. 2002. TR surfaces and conformations required to bind nuclear receptor corepressor. Mol. Endocrinol. 16:271-286.

32. Fondell, J.D., Guermah, M., Mali, S., and West, B.L. 1999. Thyroid hormone receptor-associated proteins and general positive cofactors mediate thyroid hormone receptor function in the absence of the TATA box-binding protein-associated factors of TFIID. Proc. Natl. Acad. Sci. U. S. A. 96:1959-1964.

33. Onate, S.A., Tsai, S.Y., Tsai, M.J., and O'Malley, B. 1995. Sequence and characterization of a coactivator for the steroid hormone receptor superfamily. Science. 270:1354-1357.

34. Xu, J., et al. 1998. Partial hormone resistance in mice with disruption of the steroid receptor coactivator-1 (SRC-1) gene. Science. 279:1922-1925.

35. Weiss, R.E., Xu, J., Ning, G.J.P., O'Malley, P., and Refetoff, S. 1999. Mice deficient in the steroid receptor co-activator 1 (SRC-1) are resistant to thyroid hormone. EMBO J. 18:1900-1904.

36. Xu, W., et al. 2001. A transcriptional switch mediated by cofactor methylation. Science. 294:2507-2511.

37. Treuter, E., Albrektsen, T., Johansson, L., Leers, J., and Gustafsson, J.A. 1998. A regulatory role for RIP140 in nuclear receptor activation. Mol. Endocrinol. 12:864-881.

38. Fernandes, I., et al. 2003. Ligand-dependent nuclear receptor corepressor LCoR functions by histone deacetylase-dependent and -independent mechanisms. Mol. Cell. 11:139-150.

39. Vo, N., Fjeld, C., and Goodman, R.H. 2001. Acetylation of nuclear hormone receptor-interacting protein RIP140 regulates binding of the transcriptional corepressor CtBP. Mol. Cell. Biol. 18:6181-6188.

40. Kumar, V., et al. 2002. Transcription corepressor $\mathrm{CtBP}$ is an $\mathrm{NAD}(+)$-regulated dehydrogenase. Mol. Cell. 10:857-869.

41. Safer, J., Cohen, R.C., Hollenberg, A.N., and Wondisford, F.E. 1998. Defective release of corepressor by hinge mutants of the thyroid hormone receptor found in patients with resistance to thyroid hormone. J. Biol. Chem. 273:30175-30182.

42. Oberste-Berghaus, C., et al. 2000. Thyroid hormone-independent interaction between the thyroid hormone receptor beta2 amino terminus and coactivators. J. Biol. Chem. 21:1787-1792.

43. Hollenberg, A.N., Moden, T., Madura, J.P., Lee, K., and Wondisford, F.E. 1996. Function of nuclear co-repressor protein on thyroid hormone response elements is regulated by the receptor A/B domain. J. Biol. Chem. 45:28516-28520.

44. Pohlenz, J., et al. 1999. Improved radioimmunoassay for measurement of mouse thyrotropin in serum: strain differences in thyrotropin concentration and thyrotroph sensitivity to thyroid hormone. Thyroid. 9:1265-1271. 